\title{
Relación entre las capturas de Megapitaria squalida (Bivalvia: Veneridae) y la temperatura superficial del mar en la Bahía de la Paz, Baja California Sur, México
}

\author{
Mariana Vázquez Hurtado슬 Marlenne Manzano Sarabia ${ }^{2}$ \& Alfredo Ortega Rubio ${ }^{1}$ \\ 1. Centro de Investigaciones Biológicas del Noroeste, S.C., Mar Bermejo No. 195. Colonia Playa Palo de Santa Rita. La \\ Paz, Baja California Sur. México; mvazquez@cibnor.mx, aortega@cibnor.mx \\ 2. Facultad de Ciencias del Mar, Universidad Autónoma de Sinaloa. Paseo Claussen s/n. A.P. 610. Mazatlán, Sinaloa. \\ México; mmanzano@uas.uasnet.mx
}

Recibido 01-II-2010. Corregido 05-VIII-2010. Aceptado 02-IX-2010.

\begin{abstract}
Megapitaria squalida (Bivalvia: Veneridae) fishery landings and temperature relationship in Bahía de la Paz, México. The clam Megapitaria squalida is a fishing resource with increasing importance in Northwestern Mexico. Nevertheless, this fishery has shown important variations that could be related to environmental factors. To assess this, monthly landings of M. squalida were analyzed during 2002-2005 for Bahía de La Paz, B.C.S., and were related with monthly time series of sea surface temperature, derived from MODIS-Aqua Sensor. The results showed a positive and significant relationship between sea surface temperature and clam landings. The likely impact of anomalous conditions of sea temperature on this resource is discussed. Rev. Biol. Trop. 59 (1): 151-157. Epub 2011 March 01.
\end{abstract}

Key words: Megapitaria squalida, commercial landings, chocolate clam, sea surface temperature, Baja California Sur, Mexico.

Los ecosistemas costeros son estudiados en sus diferentes componentes debido a su importancia socio-económica y ecológica. Sin embargo, marismas, manglares, humedales, playas de arena, arrecifes de coral y deltas de ríos son particularmente vulnerables tanto a factores ambientales como humanos (IPCC 1995).

La almeja chocolate Megapitaria squalida (Sowerby 1835), es uno de los bivalvos más abundantes en el noroeste de México (Arellano et al. 2006). El recurso "almeja chocolate" está conformado por dos especies del género Megapitaria spp. (Familia Veneridae, Sub-familia Pitarinae): $M$. aurantiaca conocida como "almeja roja" y M. squalida o "almeja negra o chocolate". La primera de éstas comprende poblaciones aisladas y muy escasas que habitan aguas profundas; la segunda es mucho más abundante en aguas con menor profundidad, con fondo arenoso (Singh et al. 1991, Baqueiro \&Aldana 2003). La distribución de Megapitaria spp. ha sido reportada desde la Laguna Ojo de Liebre, Baja California Sur, México hasta Mancora, Perú, (Baqueiro \& Stuardo 1977). En la Bahía de la Paz, B.C.S., M. squalida representa un recurso con una alta demanda en el mercado regional (Singh et al. 1991) y ha sido estudiada en diferentes aspectos. Baqueiro \& Stuardo (1977) realizaron un estudio sobre la biología, ecología y explotación de dos especies de Megapitaria spp. en la Bahía de la Paz, mientras que Baqueiro (1978) estableció la distribución de dos especies de Megapitaria spp. con base a la granulometría del sedimento en la Bahía de Zihuatanejo, Guerrero. Asimismo, en la Bahía de la Paz B.C.S., Singh et al. (1991) realizaron un estudio poblacional 
en Punta Coyote, mientras que Mazón et al. (1994) implementaron un cultivo en suspensión y en fondo de $M$. squalida. Recientemente, Arellano et al. (2006) y Schweers et al. (2006) realizaron estudios sobre el patrón reproductivo y dinámica poblacional de $M$. squalida en Baja California Sur, respectivamente. Schweers et al. (2006) determinaron que la densidad de población es de $2.01 \pm 1.41 \mathrm{ind} / \mathrm{m}^{2}$ en Bahía Magdalena, sin embargo no hay estudios que determinen este parámetro en cada uno de los lugares en los que se captura el recurso en la Bahía de la Paz.

Aunque, M. squalida es usualmente considerada como una especie con bajo valor comercial, la pesquería de esta almeja en el noroeste de México se ha intensificado y la especie está siendo capturada como un recurso alternativo, cuando las principales especies comerciales no están disponibles por las restricciones del esfuerzo pesquero. La necesidad de proponer recomendaciones para el manejo de especies de moluscos bivalvos, ha motivado el estudio de tendencias en el comportamiento de las poblaciones y su biología reproductiva, con relación a las características ambientales de los hábitats en donde son explotadas (Baqueiro \& Aldana 2003).

La variabilidad ambiental es reconocida como un factor importante que influye en la distribución y abundancia de los recursos marinos. Del Monte \& Lluch-Belda (2004) resaltan la importancia de la variabilidad -natural o inducida- de las condiciones físicas del ambiente, ya que los cambios en éste pueden llevar a las poblaciones a niveles a partir de los cuales ya no pueden recuperarse. Los factores que determinan el comportamiento poblacional y reproductivo en los moluscos en las diferentes zonas son de variada naturaleza. Baqueiro \& Aldana (2003) mencionan que la capacidad reproductiva de los moluscos bivalvos está determinada genéticamente con fuerte influencia del medio ambiente a través de la temperatura, intensidad y duración de la luz, determinando la época y éxito de reproducción. En este sentido, la temperatura del océano es una variable ambiental de importancia, ya que además afecta la distribución y abundancia de las poblaciones en diferentes escalas espaciales y temporales, así como algunos de sus parámetros poblacionales. Aunque la almeja chocolate se extrae de forma manual y por medio de buceo libre, la pesquería ha tenido un auge en los últimos años en el noroeste de México, por lo que se requiere conocer el comportamiento del recurso ante fluctuaciones ambientales (Baqueiro \& Aldana 2003). El objetivo del presente estudio es analizar las variaciones en los volúmenes de captura de almeja chocolate en relación con la variabilidad de la temperatura superficial del mar durante el período 20022005 en la Bahía de la Paz, Baja California Sur, México.

\section{MATERIAL Y MÉTODOS}

Descripción del área de estudio: $\mathrm{La}$ Bahía de la Paz se ubica en la parte sureste de la Península de Baja California Sur, México (Fig. 1) es uno de los ecosistemas costeros más extensos del Golfo de California (Jiménez 1997). Tiene una longitud de $\sim 90 \mathrm{~km}$, anchura de $\sim 60 \mathrm{~km}$, un área aproximada de $4500 \mathrm{~km}^{2}$ y se comunica a través de dos bocas con la parte sur del Golfo de California (Jiménez 1997). Su alta productividad biológica, sus características hidrográficas y topográficas lo distinguen de la zona oceánica adyacente, confiriéndole un régimen oceanográfico propio (Martínez et al. 2006).

A partir de la información oficial obtenida de las capturas (estadísticas de desembarques), se analizaron y estandarizaron datos mensuales de volumen de producción de $M$. squalida durante el período 2002-2005 registrados por la Subdelegación de Pesca de la Delegación de la Secretaría de Agricultura, Ganadería, Desarrollo Rural, Pesca y Alimentación en Baja California Sur, México. Mediante investigación bibliográfica, se identificaron los periodos de desove y se comparó con la temporada de pesca a partir de los datos de captura registrados. Los datos de captura provienen de 3 localidades de profundidad somera (3-10m) de fondo arenoso. En estos sitios los individuos de 


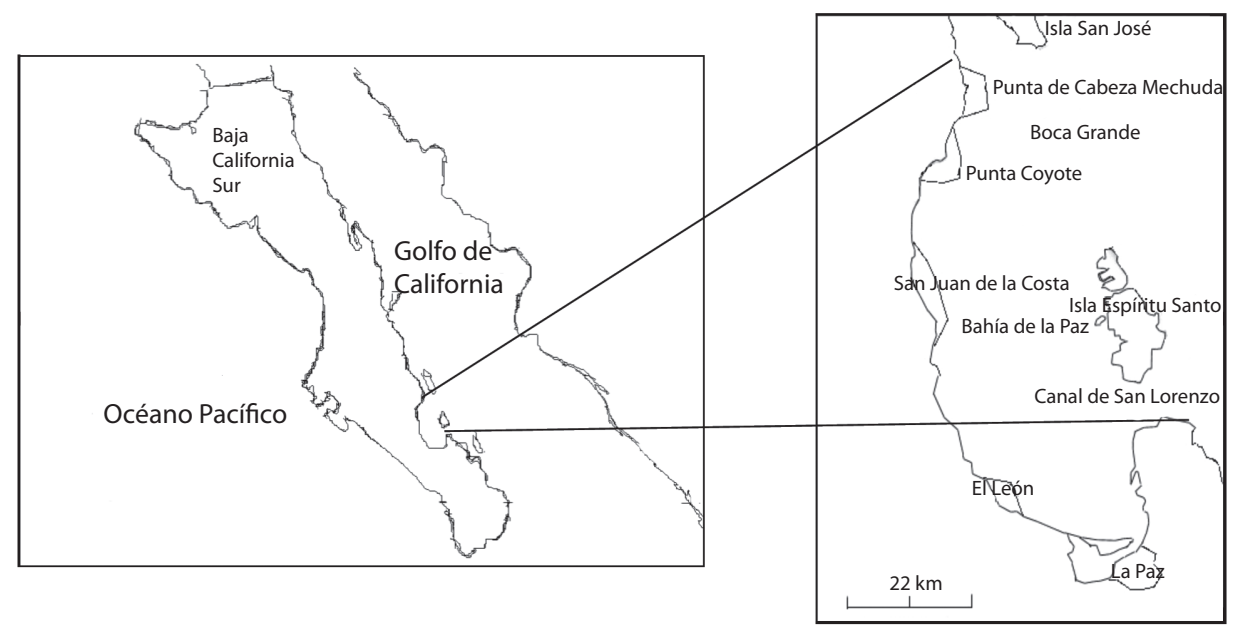

Fig. 1. Bahía de la Paz, Baja California Sur, México y principales zonas de captura de M. squalida.

Fig. 1. La Paz Bay, South Baja California, Mexico and main captures zones of M. squalida.

esta especie son capturados a mano por buzos artesanales por medio de buceo autónomo, por lo que no existe una evaluación de la densidad de población en los 3 sitios identificados. Se construyeron series de tiempo de la temperatura superficial del mar (TSM) a partir de imágenes mensuales de $1 \mathrm{~km}$ de resolución de pixel, derivadas del sensor MODIS-Aqua y fueron analizadas con el programa WIM ${ }^{\mathrm{MR}}$ (http:// www.wimsoft.com). Asimismo, se realizó una estimación del ciclo estacional de la TSM y se calcularon las anomalías mensuales a partir de la diferencia de la imagen en el mes $i$ respecto a la imagen promedio general de ese mes en el periodo de estudio. Se realizó un análisis de correlación lineal simple entre las capturas estandarizadas y la serie de anomalías mensuales de la TSM con el programa estadístico Statistica 6.0.

\section{RESULTADOS}

De acuerdo con los avisos de arribo se identificó que las principales zonas de captura en la Bahía de la Paz son: Punta Cabeza Mechuda, El Mechudo, San Juan de la Costa, destacando Punta Coyote $\left(24^{\circ}-41^{\prime}\right.$ N $-110^{\circ}-42^{\prime}$
W) con los mayores volúmenes de captura registrados y representando el $88.9 \%$ de la captura total de almeja chocolate (Fig. 1, Cuadro 1). Los resultados muestran que $M$. squalida se captura a lo largo del año, con valores máximos durante los meses de febrero, marzo y abril, cuando la temperatura superficial del mar oscila entre $20^{\circ} \mathrm{C}$ y $25^{\circ} \mathrm{C}$ (Fig. 2). En esta especie, la época de desove se prolonga de mayo a diciembre, fueron observados dos periodos de desove: mayo-julio y noviembre-diciembre

\section{CUADRO 1}

Volumen de producción (ton) de Megapitaria squalida y porcentaje relativo

TABLE 1

Megapitaria squalida production (ton) and relative percentage at Bahia de la Paz.

\begin{tabular}{lcc}
\multicolumn{1}{c}{ Localidad } & \multicolumn{2}{c}{$2002-2005$} \\
& $\begin{array}{c}\text { Volumen de } \\
\text { captura (ton) }\end{array}$ & $\begin{array}{c}\% \\
\text { Relativo }\end{array}$ \\
\hline Punta Cabeza Mechuda & 18.56 & 6.96 \\
Punta Coyote & 236.96 & 88.91 \\
San Juan de la Costa & 9.00 & 3.37 \\
El León & 2.00 & 0.75 \\
Total & 266.51 & 100
\end{tabular}




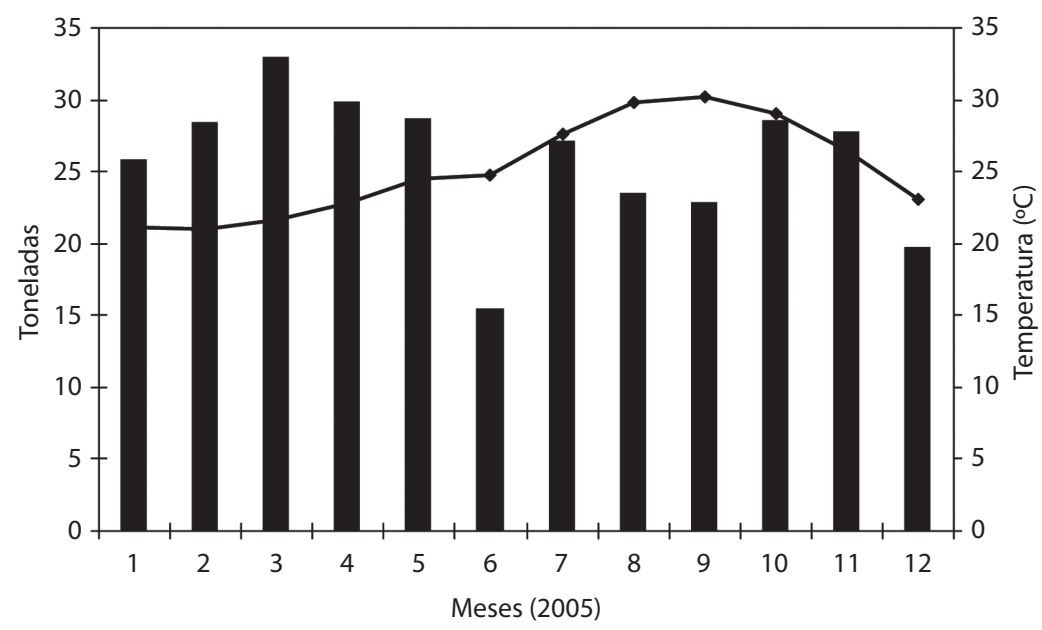

Fig. 2. Ciclo estacional (2005) de la temperatura superficial del mar en la Bahía de La Paz y producción en toneladas de $M$. squalida durante 2005.

Fig. 2. Seasonal cycle of the surface temperature of the sea in La Paz Bay and production in tons of M. squalida during 2005.

(Fig. 3), así como un periodo de máxima intensidad de desove durante los meses de agostooctubre (Singh et al. 1991). La Fig. 3 muestra que la pesca se realizó todo el año (excepto en 2002), aún en la época reproductiva.

En el periodo de estudio se observaron dos pulsos de incremento en las capturas, uno hacia el invierno de 2002-2003 y el otro en el verano de 2004, correspondientes a los meses en los que se presentaron anomalías positivas en la TSM en el intervalo 0.5 a $1^{\circ} \mathrm{C}$ (Fig. 4). Se estimó el coeficiente de correlación anual para $2002(\mathrm{r}=0.9, \mathrm{p}=0.01), 2003(\mathrm{r}=0.9, \mathrm{p}<0.01)$, $2004(\mathrm{r}=0.8, \mathrm{p}<0.01)$ y $2005(\mathrm{r}=-0.3, \mathrm{p}=>0.05)$, mientras que el coeficiente de correlación para el periodo 2002-2005 fue de 0.5 ( $\mathrm{p}<0.01)$, lo cual sugiere que existe una relación positiva y significativa entre la temperatura del mar y la captura del recurso. Asimismo, durante 2005 se observaron anomalías negativas en la TSM coincidentes con la disminución de las capturas. Las anomalías negativas de la TSM se mantuvieron desde los últimos meses de 2004 hasta 2005, siendo condiciones que pudieron afectar algunos parámetros reproductivos y/o de mortalidad de M. squalida.

\section{DISCUSIÓN}

La almeja chocolate es un recurso que tiene gran importancia para los pescadores artesanales de la región. De acuerdo con la información oficial obtenida (avisos de arribo), se determinó que la zona de captura con mayor abundancia es Punta Coyote, ubicada en la costa norte de la Bahía de la Paz y que se caracteriza por presentar fondo arenoso, con pendiente poco pronunciada y una profundidad que varía entre los 4 y los 9m, la cual favorece el desarrollo de la almeja chocolate y facilita por consiguiente su extracción (Singh et al. 1991).

Kennedy \& Mihursky (1972) mencionan que las condiciones de mayor temperatura someten a los organismos bivalvos a situaciones de estrés alterando el metabolismo respiratorio y su capacidad de adaptación. Asimismo, 


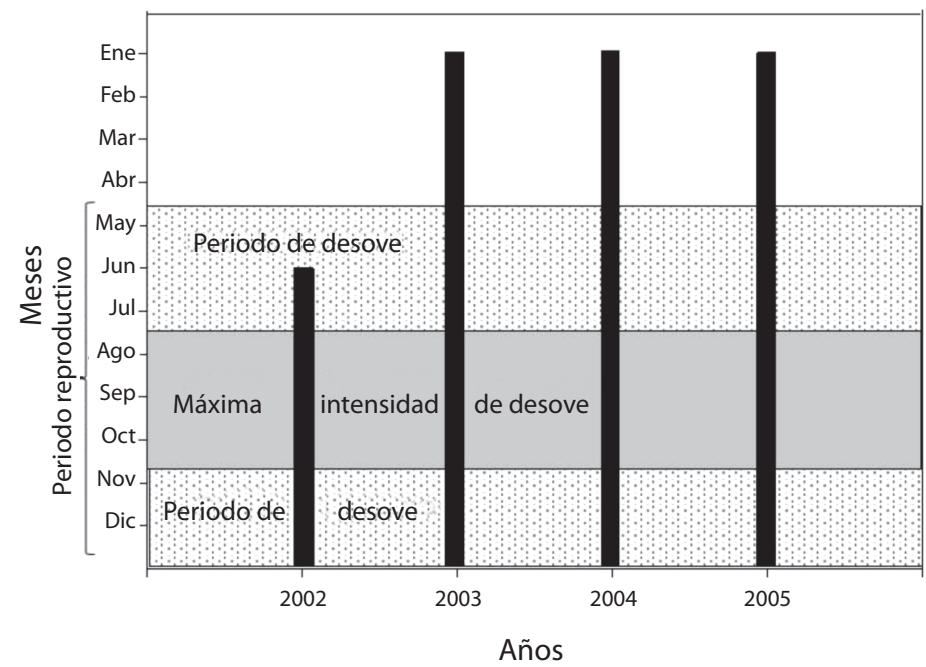

Fig. 3. Temporada de pesca anual (barras negras) de almeja chocolate Megapitaria squalida ${ }^{1}$ y período reproductivo ${ }^{2}$ : Período de desove y máxima intensidad de desove. Fuentes: 1. Delegación Estatal de la SAGARPA en Baja California Sur. 2. Singh et al. 1991.

Fig. 3. Season of annual fishing (I sweep blacks) of chocolate clam Megapitaria squalida ${ }^{1}$ and reproductive period ${ }^{2}$ : Period of egg-laying and maximum intensity of egg-laying. Sources: 1. State delegation of SAGARPA in South Baja California. 2. Singh et al., 1991.

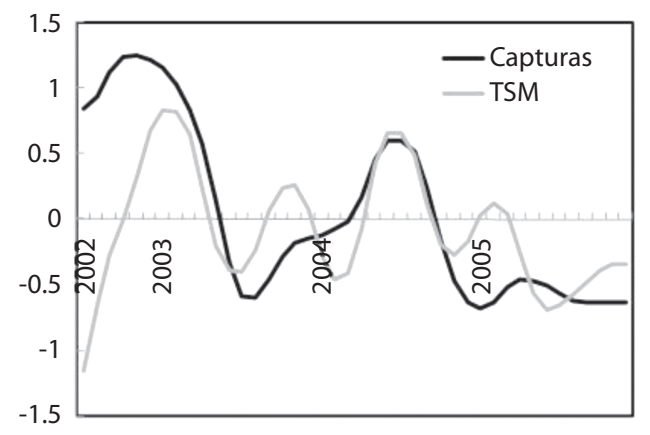

Fig. 4. Capturas estandarizadas de Megapitaria squalida y anomalías de temperatura superficial del mar durante el periodo 2002-2005.

Fig. 4. Captures standardized of Megapitaria squalida and anomalies of surface temperature of the sea during period 2002-2005.

la temperatura en los moluscos bivalvos es un factor ambiental importante que influye de manera significativa en el proceso de reproducción (e.g. gametogénesis y desove; Arellano et al. 2006). En la Bahía de la Paz el ciclo anual de la TSM presenta dos estaciones bien definidas relacionadas con el patrón estacional de vientos (Martínez et al. 2006). Los valores promedio de TSM más altos se registraron durante los meses de agosto, septiembre y octubre durante el período de estudio $\left(29.7,30.2\right.$ y $28.9^{\circ} \mathrm{C}$, respectivamente) lo cual coincide con lo reportado por Martínez et al. (2006). Los mayores valores de captura fueron observados en el intervalo de $20^{\circ} \mathrm{C}$ y $25^{\circ} \mathrm{C}$ (otoño-invierno). Por otra parte, el patrón reproductivo de la almeja $M$. squalida reportado por Singh et al. (1991) sugiere que el periodo de máxima intensidad de desove se realiza durante los meses de agosto a octubre, correspondiendo con el periodo que registró los mayores valores promedio de temperatura a lo largo del año en el presente estudio, lo que indica que el aumento en la TSM estimula los desoves de M. squalida como mencionan Arellano et al. (2006).

Durante el periodo de estudio (2002-2005) se observó una correlación positiva y significativa entre la captura de $M$. squalida y las variaciones (anomalías) en la TSM (Fig. 4). 
Aunque estos resultados sugieren una tolerancia de $M$. squalida a condiciones cálidas en la TSM, se desconoce el posible impacto de eventos climáticos de gran magnitud como El Niño Oscilación del Sur. Como ha sido discutido por diversos autores (e.g. Soto et al. 1999, Lavín et al. 2003, Thatje et al. 2008), los eventos El Niño afectan las condiciones oceánicas-atmosféricas del Golfo de California y en consecuencia en los diferentes componentes bióticos de este Gran Ecosistema Marino (Lluch et al. 2007). Mazón et al. (1994) reportaron que en condiciones elevadas de temperatura superficial del mar $\left(32^{\circ} \mathrm{C}\right)$ se observó un $10 \%$ de sobrevivencia de organismos cultivados en suspensión. Es posible que episodios cálidos sostenidos y de mayor intensidad (e.g. eventos El Niño) puedan afectar las capturas de $M$. squalida, así que se requieren estudios sobre la vulnerabilidad de esta especie a tales eventos. Por otra parte, se observó una disminución en las capturas de $M$. squalida en condiciones frías (Fig. 4) por lo que es posible que exista un impacto significativo durante condiciones tipo La Niña. De acuerdo con el Índice Multivariado de El Niño (http://www.esrl.noaa.gov/psd/ data/climateindices/list/) el periodo de estudio considerado fue dominado por una fase cálida moderada, por lo que no fue posible determinar el comportamiento de las capturas ante un evento frío/cálido de gran magnitud (e.g. El Niño 1997-1998).

De acuerdo con los resultados derivados del presente estudio, se determinó que la temperatura superficial del mar es un factor ambiental importante en la variación de las capturas así como en el inicio de los desoves de $M$. squalida; sin embargo, se sugiere investigar los intervalos de tolerancia térmica de $M$. squalida y su efecto en su fisiología reproductiva, ya que de esta forma se podrá determinar la vulnerabilidad del recurso ante diferentes escenarios de variabilidad ambiental, principalmente en condiciones tipo El Niño/La Niña, de utilidad para proponer medidas de manejo y administración sustentable del recurso.

\section{AGRADECIMIENTOS}

Se agradece al Proyecto CONACyTSEMARNAT 2008-CO1-107923 y CONACyT-Retención-117014 el apoyo otorgado, al Ocean Biology Processing Group de la NASA y a Mati Kahru (Scripps, UCSD) por proporcionar las imágenes de satélite utilizadas para obtener las anomalías de la temperatura superficial del mar en este estudio. Asimismo, los autores agradecen a dos revisores anónimos y a los Editores de la Revista de Biología Tropical, por el tiempo y esfuerzo dedicados a mejorar una primera versión de nuestro manuscrito.

\section{RESUMEN}

La almeja chocolate Megapitaria squalida es un recurso pesquero con creciente importancia en el noroeste de México al registrarse un incremento en los volúmenes de captura. Sin embargo, esta pesquería ha observado variaciones importantes en las capturas que podrían estar relacionadas con factores ambientales. Se obtuvieron datos mensuales de producción de M. squalida durante 20022005 y se estimaron series de tiempo de temperatura superficial del mar en la Bahía de La Paz B.C.S. a partir de imágenes mensuales derivadas del sensor MODIS-Aqua. Los resultados indican una relación positiva significativa entre la temperatura superficial del mar y los volúmenes de captura, razón por la cual nuestro trabajo tiene como objetivo estudiar las relaciones entre capturas de Megapitaria squalida (Bivalvia: Veneridae) y la temperatura superficial del mar en La Bahía de la Paz, Baja California Sur, México.

Palabras clave: Megapitaria squalida, capturas comerciales, almeja chocolate, temperatura superficial del mar, Baja California Sur, México.

\section{REFERENCIAS}

Arellano, M.M., A.M. Quiñones, V.B.P. Ceballos \& F.M. Villalejo. 2006. Reproductive pattern of the squalid callista Megapitaria squalida from Northwestern Mexico. J. Shellfish Res. 25: 849-855.

Baqueiro, E. \& J. Stuardo. 1977. Observaciones sobre la biología, ecología y explotación de Megapitaria aurantiaca (Sowerby 1831), M. squalida (Sowerby 1835) y Dosinia ponderosa (Gray 1838) (Bivalvia: Veneridae) de la Bahía de Zihuatanejo e Isla Ixtapa, Gro., México. An. Cent. Cienc. Mar Limnol. 4: 161-208. 
Baqueiro, E. 1978. Sobre la distribución de Megapitaria aurantiaca (Sowerby), M. squalida (Sowerby) y Dosinia ponderosa (Gray) en relación a la granulometría del sedimento (Bivalvia: Veneridae): Nota Científica. An. Cent. Cienc. Mar Limnol. 6: 25-31.

Baqueiro, E. \& D. Aldana. 2003. Patrones en la biología poblacional de moluscos de importancia comercial en México. Rev. Biol. Trop. 51: 97-107.

Del Monte, L.P. \& D. Lluch-Belda. 2004. Reflexiones sobre la explotación, recuperación y extinción de poblaciones naturales. Interciencia 29: 158-162.

IPCC. 1995. Informe del grupo intergubernamental de expertos sobre el cambio climático, Segunda Evaluación. IPCC, Ginebra, Suiza.

Jiménez, I.A., M.N. Obeso \& D.S. de León. 1997. Oceanografía física de la Bahía de la Paz, B.C.S., p. 31-41. In R.J. Urbán \& M.R. Ramírez (eds). La Bahía de La Paz, investigación y conservación. UABCSCICIMAR-SCRIPPS. La Paz, Baja California Sur, México.

Kennedy, V.S. \& J.A. Mihursky. 1972. Effects of temperature on the respiratory metabolism of three Chesapeake Bay bivalves. Chesap. Sci. 13: 1-22.

Lavín, L.F., E.H. Palacios \& C. Cabrera. 2003. Sea surface temperatura anomalies and trend in the Gulf of California. Geofisica Int. 42: 363-375.

Lluch, C.S.E., E.A.N. Aragón, F.S. Arreguín \& D.G. Aurioles. 2007. The Gulf of California: Review of ecosystem status and sustainability challenges. Progr. Oceanogr. 73: 1-26.

Martínez, F.G., D.R. Cervantes \& E.R. González. 2006. Caracterización de la temperatura superficial del mar y climatología de la Bahía de la Paz, B.C.S., México. CICIMAR Oceánides 21: 81-91.

Mazón, S.J.M., V.M. Zaragoza, M.A. Maeda, G.T. Reynoso, S.P. Monsalvo \& R.L. Morales. 1994. Cultivo en suspensión y en fondo de almeja chocolate Megapitaria squalida, en la Bahía de la Paz, Baja California Sur, México. Rev. Invest. Cien. Área Ciencias del Mar, 5 (No. Esp. AMAC): 67-71.

Schweers, T., M. Wolff, V. Koch \& F. Sinsel-Duarte. 2006. Population dynamics of Megapitaria squalida (Bivalvia: Veneridae) at Magdalena Bay, Baja California Sur, Mexico. Rev. Biol. Trop. 54: 1003-1017.

Singh, C.J., B.J.A. Vélez \& L.M.C. Fajardo. 1991. Estudio poblacional de la almeja chocolate Megapitaria squalida (Sowerby, 1835) en Punta Coyote, Bahía de la Paz B.C.S., México. Cien. Pesq. 8: 7-22.

Soto M.L., S.G. Marinone \& A.S Parés. 1999. Time and spatial variability of sea surface temperature in the Gulf of California. Cien. Mar. 25: 1-30.

Thatje, S., O. Heilmayer \& J. Laudien. 2008. Climate variability and El Niño Southern Oscillation: implications for natural coastal resources and management. Helgol Mar. Res. 62: S5-S14. 